\title{
LENGTHENING PRIMARY CILIA ENHANCES CELLULAR MECHANOSENSITIVITY
}

\author{
M. Spasic and C.R. Jacobs* \\ Department of Biomedical Engineering, Columbia University, New York, NY, USA
}

\begin{abstract}
The primary cilium is a mechanosensor in a variety of mammalian cell types, initiating and directing intracellular signalling cascades in response to external stimuli. When primary cilia formation is disrupted, cells have diminished mechanosensitivity and an abrogated response to mechanical stimulation. Due to this important role, we hypothesised that increasing primary cilia length would enhance the downstream response and therefore, mechanosensitivity. To test this hypothesis, we increased osteocyte primary cilia length with fenoldopam and lithium and found that cells with longer primary cilia were more mechanosensitive. Furthermore, fenoldopam treatment potentiated adenylyl cyclase activity and was able to recover primary cilia form and sensitivity in cells with impaired cilia. This work demonstrates that modulating the structure of the primary cilium directly impacts cellular mechanosensitivity. Our results implicate cilium length as a potential therapeutic target for combating numerous conditions characterised by impaired cilia function.
\end{abstract}

Keywords: Primary cilia, mechanosensitivity, fenoldopam, osteocyte.

*Address for correspondence:

Christopher R. Jacobs

Columbia University, 351 Engineering Terr.

1210 Amsterdam Ave., Mail Code 8904, New York NY 10027, USA

Telephone: +12128544460

Email: crj2111@columbia.edu

\section{Introduction}

Mechanotransduction is a critical cellular process in a variety of tissues. Endothelial cells sense blood flow and transduce the mechanical stimuli into biochemical responses to adjust blood vessel diameter ( $\mathrm{Ku}, 1997)$. Similarly, kidney epithelial cells in the collecting duct sense and respond to varying rates of urine flow (Liu et al., 2003). Bone maintenance requires mechanical stimulation to maintain balanced formation and resorption (You et al., 2008). Understanding how cells sense mechanical cues and transduce them into biochemical responses is a critical impediment for developing novel treatments for a wide variety of diseases of structural tissues.

Cell mechanotransduction has been studied through several different mechanisms. Altering cytoskeletal mechanics by disrupting actin polymerisation with cytochalasin D inhibits flow-induced osteogenic differentiation of C3H10T1/2 mesenchymal stem cells (Arnsdorf et al., 2009). Similar experiments in osteoblasts treated with cytochalasin D and exposed to fluid flow demonstrated increased cellular mechanosensitivity, as measured by prostaglandin E2 release (Malone et al., 2007; Norvell et al., 2004). Integrins couple the extracellular matrix with the cytoskeleton through focal adhesions comprised of actin-associated proteins, such as talin and vinculin, inhibition of focal adhesion dynamics, in focal adhesion kinase knockout osteoblasts, impairs the prostaglandin E2 release indicative of mechanotransduction (Castillo et al., 2012; Geiger et al., 2001). Mathematical models of osteocytes in vivo estimate that osteocyte dendritic processes may experience higher strain than the cell body (Vaughan et al., 2014). In a separate study, a transwell filter system was used to differentiate the osteocyte cell body and dendritic processes and each was separately mechanically loaded (Burra et al., 2010). It was found that the glycocalyx of the dendritic processes is critical for the formation of integrin attachments that initiate a mechanotransduction pathway resulting in the opening of hemichannels on the cell body. Gap junctions between adjacent cells are thought to contribute to mechanotransduction by mediating calcium, ATP and prostaglandin E2 intercellular signalling (Plotkin et al., 2015). Membrane deformations also play a significant role in cell mechanosensing where stretch-activated channels, such as polycystin-2 (PC2) and transient receptor potential vanilloid 4 (TRPV4), mediate calcium influx to initiate mechanotransduction (Lee et al., 2015b; Phan et al., 2009; Piperi, 2015).

In this work we focus on primary cilia, single immotile organelles extending from the surface of nearly all mammalian cells, which have been implicated as mechanosensors in a variety of cell types. Praetorius and Spring first demonstrated that kidney epithelial cells 
respond to fluid flow and specifically that this mechanical stimulation causes primary cilia deflection (Praetorius and Spring, 2001). Furthermore, fluid flow initiates an intracellular calcium increase that is diminished when cilia are removed (Praetorius and Spring, 2003). Since then, primary cilia have been identified as mechanosensing organelles in a variety of cell types, including bone (Malone et al., 2007).

Osteocytes are mechanosensitive cells within bone, where the primary cilium functions as a mechanosensor (Malone et al., 2007). In vitro, fluid flow mechanical stimulation of osteocytes enhances expression of the osteogenic genes cyclooxygenase-2, COX-2, and osteopontin, OPN. COX-2 synthesises prostaglandin E2 and $\mathrm{OPN}$ is a critical extracellular matrix protein. Increases in the production of both are indicative of osteogenesis (Ehrlich and Lanyon, 2002; Fujihara et al., 2006; KleinNulend et al., 1997; Raisz, 1999). When osteocyte primary cilia formation is inhibited, the cells display an abrogated osteogenic response to flow, implicating the cilium as a critical mechanosensor in osteocytes (Malone et al., 2007). Furthermore, we have previously reported that adenylyl cyclases, specifically AC6, play a significant role in osteocyte mechanosensitivity (Kwon et al., 2010). Adenylyl cyclases convert ATP to the ubiquitous second messenger cAMP, a process which can be specifically stimulated by forskolin.

Despite prior in vitro work, the role of osteocyte primary cilia in vivo is yet unclear. The presence of primary cilia within mineralised bone has been addressed with conflicting reports. Tonna and Lampen found that less than $4 \%$ of osteocytes possessed primary cilia, while Uzbekov et al. reported more than $94 \%$ incidence (Tonna and Lampen, 1972; Uzbekov et al., 2012). Furthermore, the lacunar space within which osteocytes reside suggests that primary cilia are less than $1 \mu \mathrm{m}$ long, as opposed to in vitro lengths, which can be $4 \mu \mathrm{m}$ (Malone et al., 2007; McNamara et al., 2009). A recent fluid-structure interface model was developed to estimate how primary cilia may deform in vivo (Vaughan et al., 2014). It was concluded that a short cilium, approximately $0.2 \mu \mathrm{m}$, was not long enough to be a mechanosensor, but that an elongated cilium, spanning the full pericellular space, could be. We have previously utilised a mouse model where primary cilia were deleted from osteocytes and osteoblasts, resulting in impaired mechanotransduction and abrogated load-induced bone formation (Temiyasathit et al., 2012). While in vivo the specific orientation and even incidence of osteocyte primary cilia remains unclear, evidence suggests that the cilium may yet play a key role in bone mechanosensing and that modifying cilium structure may alter mechanotransduction.

While several small molecules exist to increase cilia length, in this work we utilise two drugs that are both clinically approved and have distinct mechanisms of action - fenoldopam, used to treat hypertension, and lithium, a treatment for bipolar disorder - to increase primary cilia length. Fenoldopam is a dopamine D1-like receptor agonist and has previously been used to increase primary cilia length in endothelial cells and kidney epithelial cells, potentially through an adenylyl cyclase-cAMP mechanism
(Kathem et al., 2014; Upadhyay et al., 2014). In the context of bone, MC3T3 osteoblasts express dopamine receptors $1-5$, yet the role of specific dopamine receptors and the effects of fenoldopam treatment is unknown and has never been examined in MLO-Y4 osteocytes (Lee et al., 2015a). Analysis of gene expression patterns after tibial loading in adult rats reveals a wide array of upregulated genes, including dopamine D1 receptor mRNA (Mantila Roosa et al., 2011). Interestingly, transgenic mice with a global deletion of this receptor have no apparent difference in bone architecture or calcification compared to controls (Drago et al., 1994). Mice with a homozygous deletion of the dopamine transporter have diminished bone mass, but the role of specific dopamine receptors was not examined (Bliziotes et al., 2000). Lithium is regularly used as an agonist of the Wnt signalling pathway in various cell types, including MLO-Y4 osteocytes, and has been shown to increase primary cilia length in cultured fibroblasts and neurons through a yet incompletely characterised mechanism (Bivi et al., 2013; Miyoshi et al., 2009). In Lrp5 knockout mice, lithium treatment restores bone metabolism and bone mass and activates Wnt signalling in isolated calvarial osteoblasts (Clément-Lacroix et al., 2005). Lithium and fenoldopam have distinct mechanisms of action and both increase primary cilia length in a variety of cell types, but their effects on primary cilia-mediated mechanotransduction in osteocytes has not been studied.

Due to the significance of primary cilia in cellular mechanotransduction, we hypothesised that increasing their length would enhance mechanosensitivity. Here, we treat osteocytes with lithium and fenoldopam to increase primary cilia length and then mechanically stimulate the cells. We then examine the potential of targeting primary cilia length to recover impaired primary cilia-mediated mechanotransduction using models of impaired cilia and ciliary proteins. Our results highlight the importance of cilium length in cellular mechanosensitivity and that this is a process that can be modulated by pharmacologic intervention.

\section{Materials and Methods}

\section{Cell culture and drug treatments}

MLO-Y4 osteocytes were cultured on collagen I-coated dishes (Corning) in aMEM (Life Technologies) supplemented with $5 \%$ foetal bovine serum, $5 \%$ calf serum and $1 \%$ penicillin/streptomycin at $37{ }^{\circ} \mathrm{C}$ and $5 \% \mathrm{CO}_{2}$. Fenoldopam mesylate (Sigma) was used at $10 \mu \mathrm{M}$ diluted in DMSO (dimethyl sulfoxide, Sigma) and normal culture media, as previously described (Kathem et al., 2014; Upadhyay et al., 2014). Lithium chloride (Sigma) was used at $500 \mu \mathrm{M}$ diluted in normal culture media - a dose response from $50 \mu \mathrm{M}$ to $10 \mathrm{mM}$ was examined with $500 \mu \mathrm{M}$ being the lowest dose to increase length significantly (data not shown). These agents, or their vehicle control, were applied to cells for $16 \mathrm{~h}$ prior to experimentation. MTT, (methylthiazolyldiphenyltetrazolium bromide) assay (Sigma) was performed according to manufacturer's protocol to assess cell viability during drug treatments. Phase contrast microscopy with an 
Olympus CKX41 inverted microscope and 40× objective was used to assess cell morphology.

\section{Immunocytochemistry}

For primary cilia imaging and analysis, cells cultured on collagen I-coated glass were fixed in $10 \%$ formalin and treated with anti-acetylated $\alpha$-tubulin primary antibody, 1: 1, from a C3B9 hybridoma cell line (Sigma). Cilia were visualised with Alexa-Fluor 488 secondary antibody, 1:1000 (Life Technologies) and imaged with a $100 \times$ oil objective on an Olympus Fluoview FV1000 confocal microscope. Nuclei were stained with DAPI (Life Technologies). Cilia length was analysed using Image J software.

\section{Oscillatory fluid flow}

Cells were exposed to oscillatory fluid flow as a mechanical stimulus. Cells were seeded on collagen I-coated glass slides at $\sim 2,800$ cells $/ \mathrm{cm}^{2}$ and cultured for $72 \mathrm{~h}$ before application of flow. Drug treatments were applied $16 \mathrm{~h}$ prior to experimentation. Slides were loaded into parallel plate flow chambers (dimensions: $75 \times 38 \times 0.28 \mathrm{~mm}$ ) and allowed to incubate at $37^{\circ} \mathrm{C}$ for $30 \mathrm{~min}$ prior to initiation of stimulation (Kwon et al., 2010; Lee et al., 2014; Malone et al., 2007). Flow was applied for $1 \mathrm{~h}$ at $1 \mathrm{~Hz}$ with a peak flow rate of $18.8 \mathrm{~mL} / \mathrm{min}$, providing 1 Pa peak wall shear stress.

\section{mRNA expression}

Immediately after flow, cells were washed with PBS and total mRNA was isolated using TriReagent (Sigma). Total mRNA was converted to cDNA by TaqMan reverse transcriptase (Applied Biosystems). Gene expression was analysed by quantitative real-time PCR using primers and probes (Life Technologies) for analysis of COX-2
(Mm00478374 m1), OPN (Mm00436767_m1), AC6 (Mm00475772_m1), intraflagellar transport 88, IFT88 (Mm00493675_m1) and GAPDH (4351309). Samples and standards were run in triplicate and gene expression was normalised to GAPDH endogenous control, as previously performed (Kwon et al., 2010; Lee et al., 2014).

\section{RNA interference}

Gene silencing was performed by siRNA mediated knockdown and compared to scramble siRNA control (Life Technologies). For primary cilia disruption, cells were transfected with $20 \mu \mathrm{M}$ IFT88 siRNA (5'-CCAGAAACAGATGAGGACGACCTTT-3'), AC6 siRNA (5'-CCTGCCACCTACAACAGCTCAATTA-3') or scrambled siRNA control using Lipofectamine 2000 (Life Technologies) as previously described (Kwon et al., 2010).

\section{Adenylyl cyclase activity}

Adenylyl cyclase activity was quantified by cAMP ELISA (Enzo). Cells were cultured as previously described and treated with $10 \mu \mathrm{M}$ fenoldopam for $16 \mathrm{~h}$. Cells were stimulated with $10 \mu \mathrm{M}$ forskolin (Sigma) or DMSO vehicle control for $20 \mathrm{~min}$ prior to lysis with $0.1 \mathrm{M} \mathrm{HCl}$. Cell lysate was analysed according to manufacturer's protocol and normalised to total protein quantified by BCA (Thermo Fisher). All samples and standards were run in duplicate.

\section{Analysis}

All data were analysed with one-way ANOVA followed by Bonferroni post-hoc correction. Values were reported as mean \pm SEM, with $p<0.05$ considered statistically significant. Sample size, $n$, represents biological replicates.
A

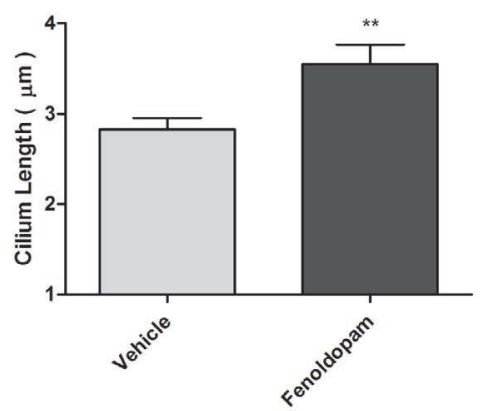

D

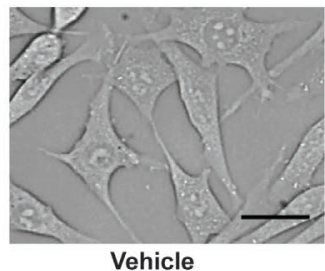

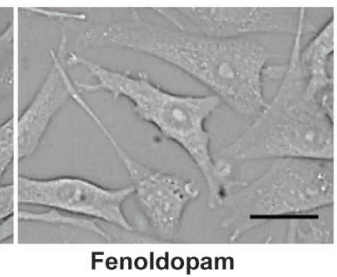

B

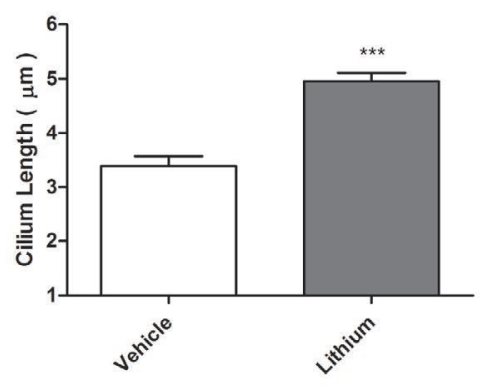

C

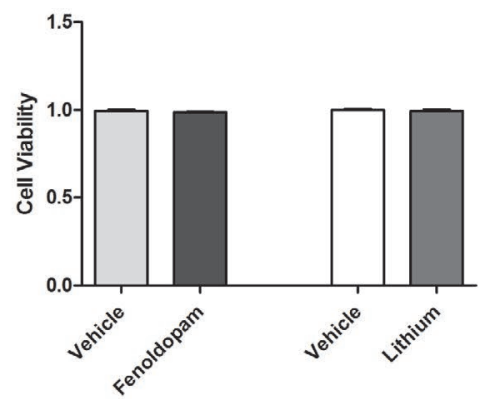

E
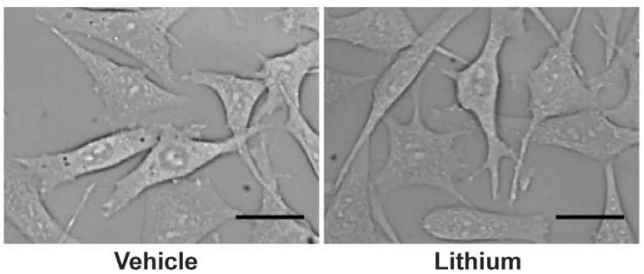

Fig. 1. Small molecule treatments increase primary cilia length. $10 \mu \mathrm{M}$ fenoldopam (A) and $500 \mu \mathrm{M}$ lithium (B) treatment for $16 \mathrm{~h}$ significantly increases primary cilia length compared to vehicle control. Drug treatments elicit no change in cell viability, as assessed by MTT assay $(\mathbf{C})$ and no gross morphological differences are exhibited (D,E). Mean \pm SEM; $n>25$ cilia for each group, $n=4$ for MTT assay; $* * p<0.01, * * * p<0.001$; scale bars $=20 \mu \mathrm{m}$. 


\section{Results}

To test our hypothesis that cilium length directly affects mechanosensitivity, we first verified that we could modulate primary cilia length. We cultured MLO-Y4 osteocytes and treated them with two distinct small molecules to increase cilia length. Cells were cultured in media supplemented with fenoldopam, lithium or vehicle control for $16 \mathrm{~h}$. Immunocytochemistry was used to image primary cilia and assess changes in cilia length. Both fenoldopam and lithium treatments induced significant increase in cilia length by $26 \% \pm 7 \%$ and $46 \% \pm 5 \%$, respectively, compared to vehicle control (Fig. 1A, B). Cell viability was assessed with MTT assay and it found no change upon drug treatments (Fig. 1C). Additionally, no gross morphological changes resulted from fenoldopam or lithium treatment (Fig. 1D, E).

Next, we examined the effect of elongating cilia on cellular mechanosensitivity by mechanically stimulating cells with longer cilia and analysing their osteogenic response. Osteocytes were treated with fenoldopam, lithium or vehicle control, and exposed to oscillatory fluid flow for $1 \mathrm{~h}$. As a control, samples were simultaneously loaded into flow chambers, but not subjected to flow. Mechanosensitivity was then quantified at the mRNA level with analysis of COX-2 and OPN expression and presented as the fold change of flow over no flow control (Fig. 2). Cells with cilia lengthened by fenoldopam were more responsive, exhibiting elevated mRNA expression of $124 \% \pm 27 \%$ and $48 \% \pm 8 \%$ of COX-2 and OPN respectively compared to unlengthened controls. Lithium resulted in a more modest, but still significant increase in response of $61 \% \pm 13 \%$ and $34 \% \pm 8 \%$ for COX-2 and OPN. This flow-induced enhanced osteogenic response was observed in cells with elongated primary cilia, regardless of the means of lengthening, suggesting that the effect is due to lengthening and not an unanticipated effect of the agents utilised.

We next sought to examine the potential of targeting primary cilia length to recover impaired cilia function. IFT88 inhibition was employed as a model of dysfunctional cilia and has previously been used to mimic the effects of polycystic kidney disease (Lehman et al., 2008). IFT88 is a critical component of intraflagellar transport and is necessary for proper primary cilia formation (Pazour et al., 2000; Yoder et al., 2002). Cells treated with IFT88 siRNA displayed decreased primary cilia length and incidence compared to scramble control (Fig. 3A, B). IFT88 siRNA treated cells were then treated with fenoldopam and cilia length and incidence were noticeably recovered (Fig.
A

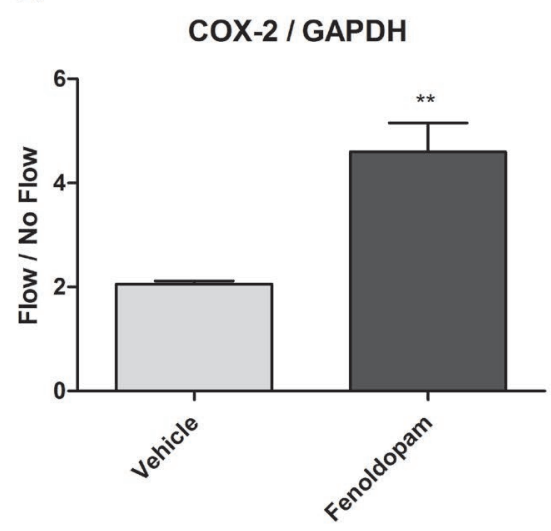

B

COX-2 / GAPDH

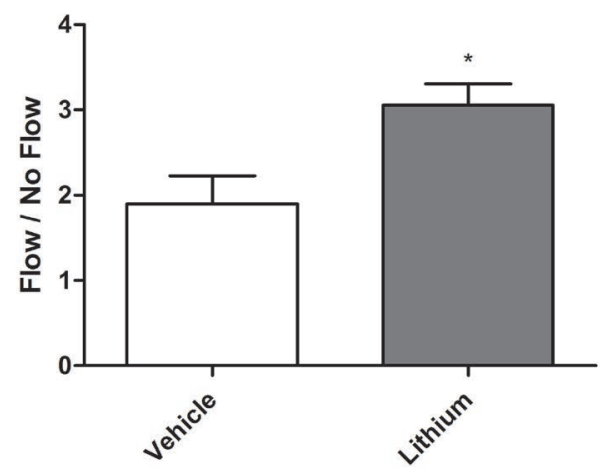

C

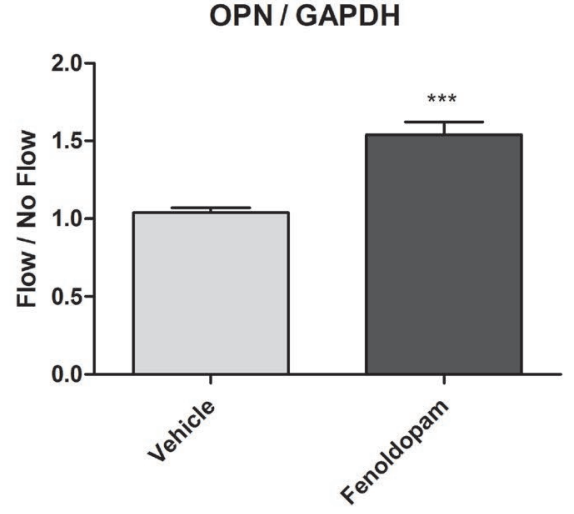

D

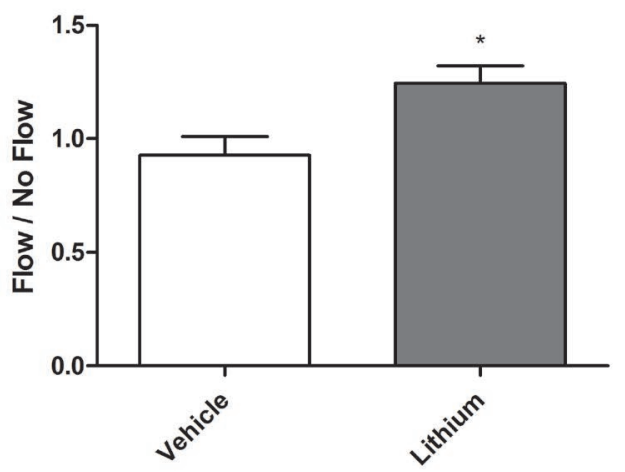

Fig. 2. Cells with longer cilia are more mechanosensitive. Cells were subjected to fluid flow for $1 \mathrm{~h}$ and the fold change of flow vs no flow control groups was compared. Cells express significant increases in COX-2 (A,B) and OPN (C,D) mRNA relative to GAPDH endogenous control when treated with either fenoldopam $(\mathbf{A}, \mathbf{C})$ or $\mathrm{LiCl}$ $(\mathbf{B}, \mathbf{D})$ for $16 \mathrm{~h}$. Mean $\pm \mathrm{SEM} ; n \geq 5$ for each group; $* p<0.05, * * p<0.01, * * * p<0.001$. 

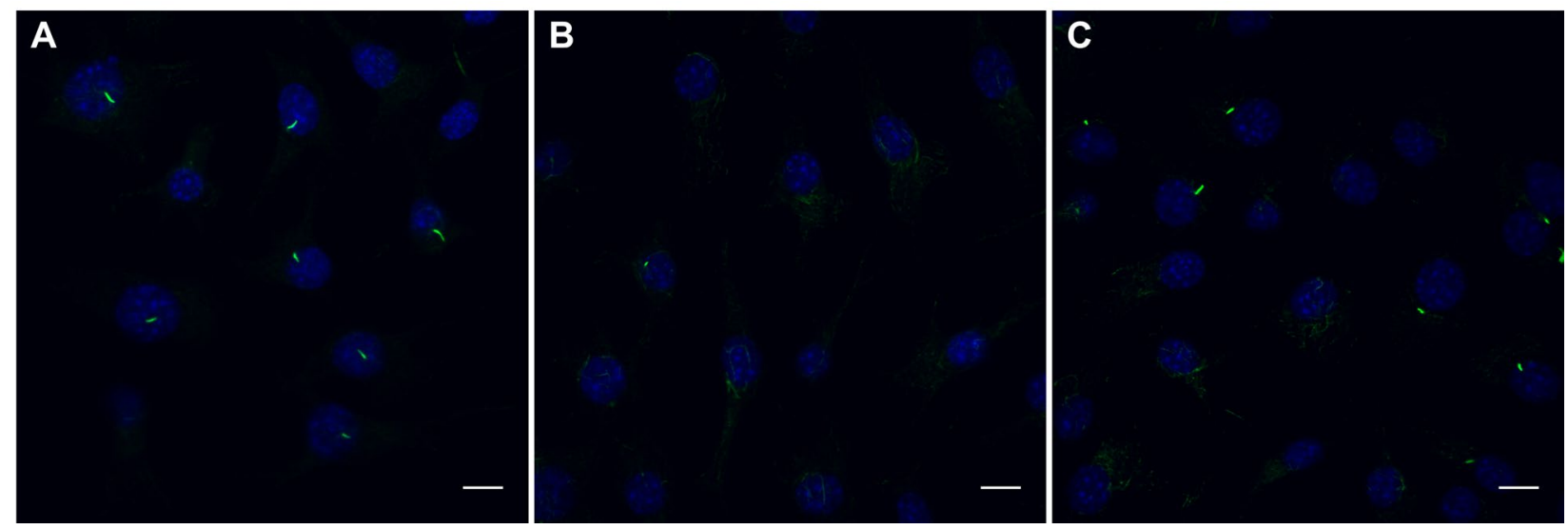

Fig. 3. Treatment with IFT88 siRNA disrupts primary cilia formation. Overlays of primary cilia (green) and nuclei (blue) illustrates primary cilia incidence. Scramble control siRNA treatment for $48 \mathrm{~h}$ does not disrupt primary cilia formation (A). IFT88 siRNA treatment results in decreased cilia length and incidence (B). Fenoldopam treatment recovers primary cilia formation in IFT88 siRNA treated cells $(\mathbf{C})$. Scale bars $=10 \mu \mathrm{m}$.

A

C

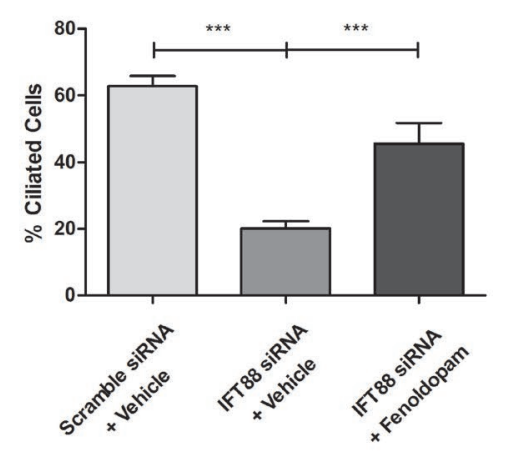

B

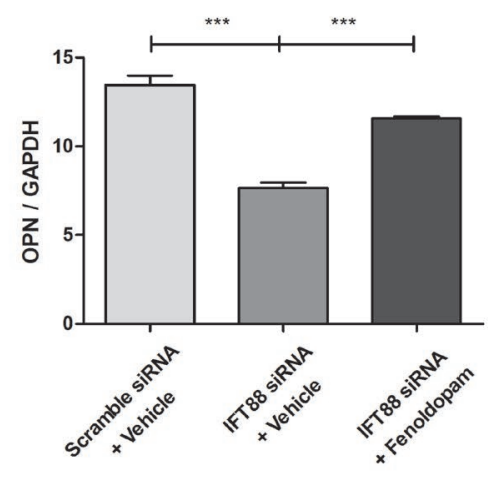

D

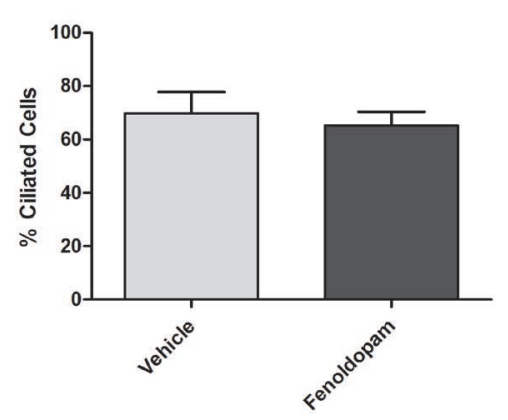

E

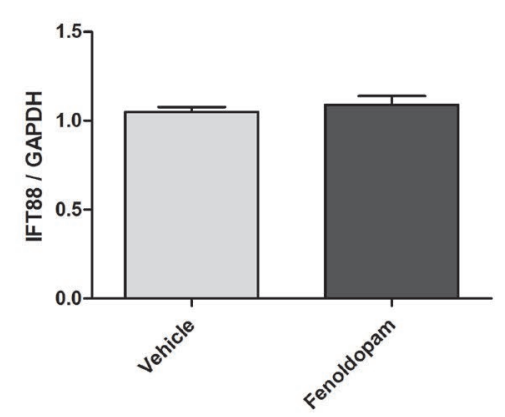

Fig. 4. Fenoldopam rescues ciliogenesis and mechanosensing. Cells treated with IFT88 siRNA have decreased cilia length compared to scramble control, while fenoldopam treatment appears to recover cilia length ( $n \geq 25$ for scramble and fenoldopam treated, $n=15$ for IFT88 siRNA alone) (A). Oscillatory fluid flow was applied to cells treated with IFT88 or scramble control siRNA. Impaired cilia display a decreased OPN response to fluid flow, while fenoldopam treatment is able to recover flow stimulated OPN expression (B). Treatment with IFT88 siRNA decreases cilia incidence, but is recovered with fenoldopam treatment; $n \geq 8$ fields of view $(\mathbf{C})$. Fenoldopam treatment on untransfected cells has no effect on cilia incidence; $n \geq 8$ fields of view (D). Fenoldopam also did not alter IFT88 mRNA expression in untransfected cells (E). Mean \pm SEM; $n \geq 4 ; * * * p<0.001$. 
A

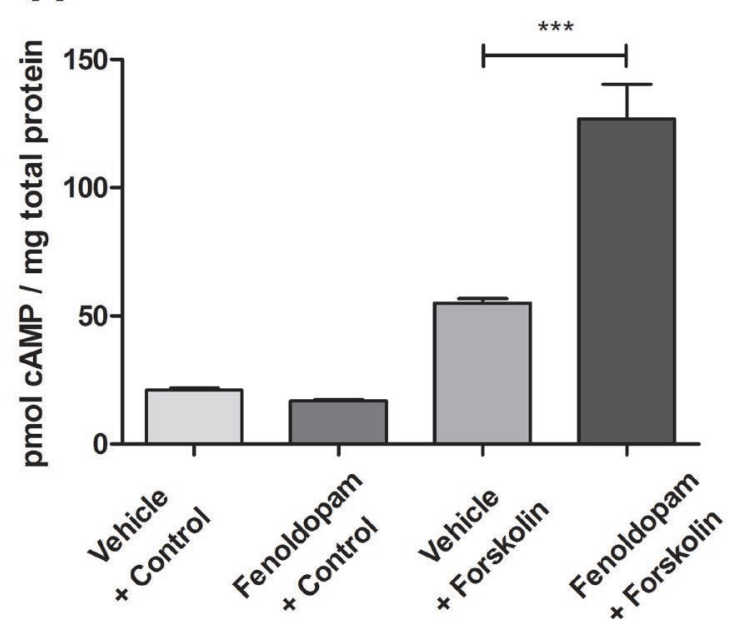

C

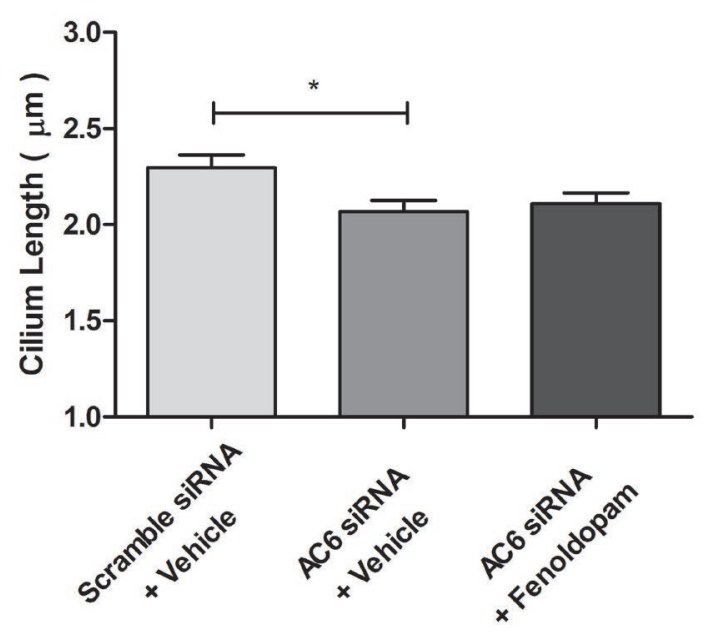

E

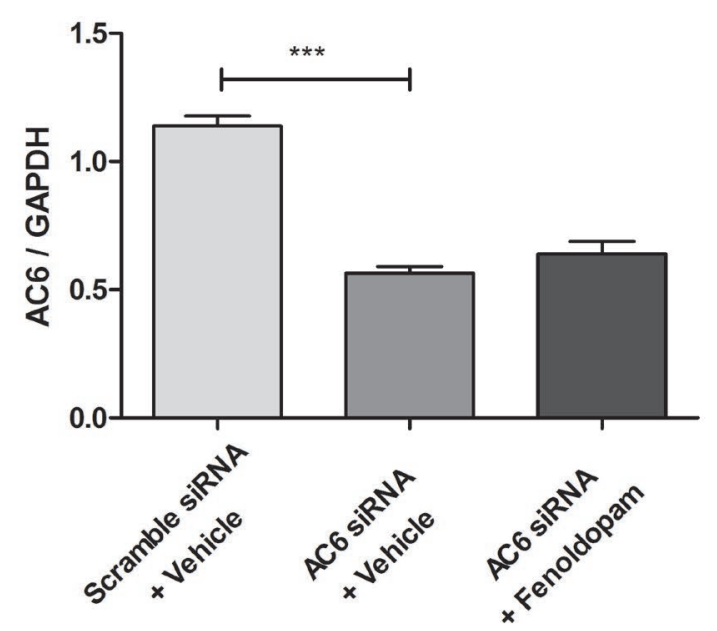

B
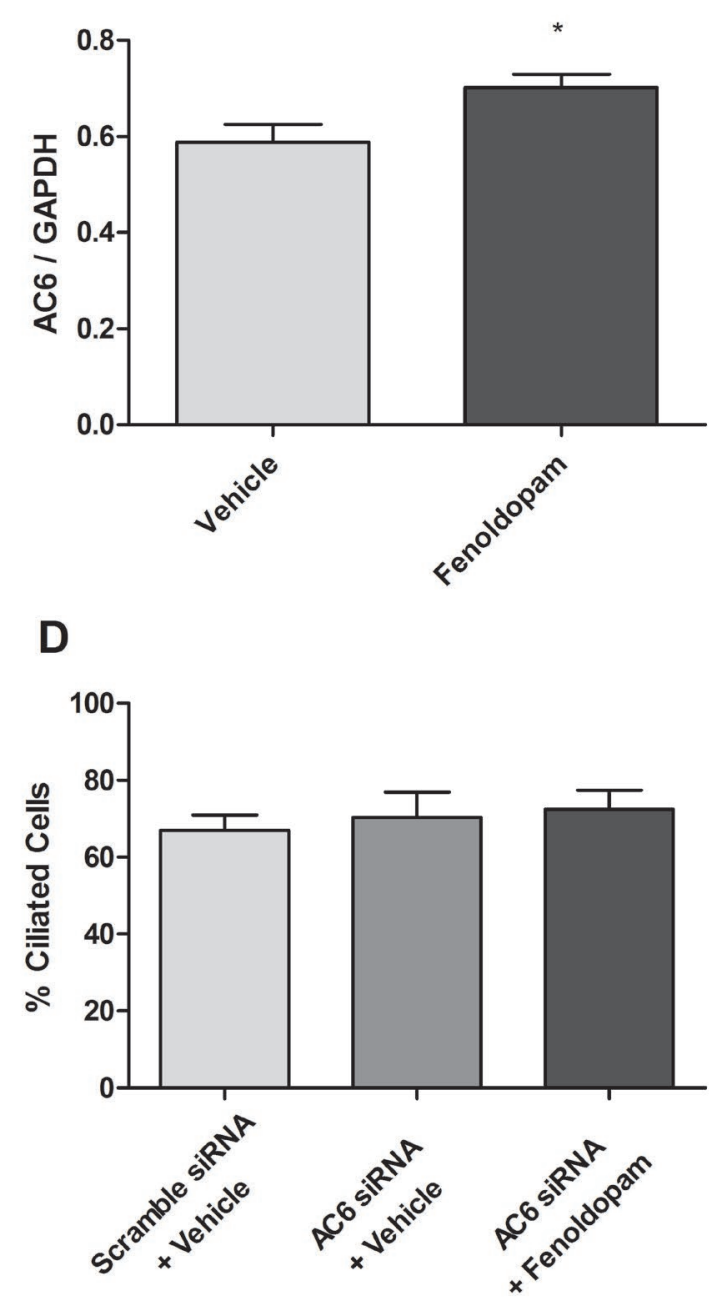

$\mathbf{F}$
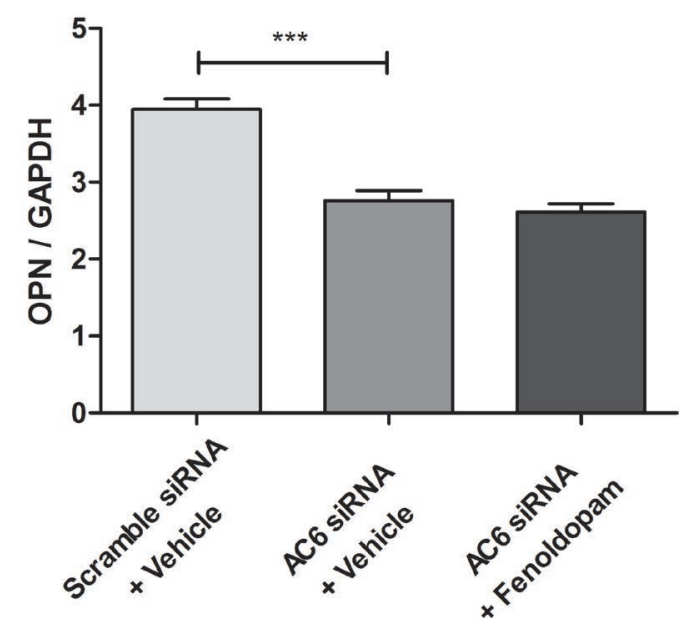

Fig. 5. Fenoldopam enhances adenylyl cyclase production and activity. Cells were treated with fenoldopam or vehicle control for $16 \mathrm{~h}$ and then forskolin stimulated for $20 \mathrm{~min}$. Fenoldopam treatment significantly increases the cAMP response to forskolin stimulation (A). Fenoldopam treatment significantly increases AC6 mRNA expression (B). AC6 knockdown decreases cilia length, but is not recovered with fenoldopam treatment $(\mathbf{C})$. Neither AC6 siRNA nor fenoldopam alter cilia incidence; $n \geq 8$ fields of view (D). Oscillatory flow applied to AC6 siRNA treated cells elicits a decrease in AC6 and OPN mRNA expression and is not recovered with fenoldopam treatment $(\mathbf{E}, \mathbf{F})$. Mean $\pm \mathrm{SEM} ; n \geq 4$ for each group; $* p<0.05,{ }^{* * *} p<0.001$. 
3C). Upon analysis, cilia of IFT88 siRNA treated cells were significantly shorter (Fig. 4A) and were present with lower incidence (Fig. 4C) than scramble control groups, with fenoldopam treatment significantly recovering cilia incidence. We then mechanically stimulated these cells to examine whether ciliogenesis recovery restored mechanosensitivity. Fluid flow was applied for $1 \mathrm{~h}$ and cells with impaired primary cilia formation displayed significantly decreased flow-induced OPN mRNA expression by $43 \% \pm 2 \%$, compared to scramble control (Fig. 4B). Then, fenoldopam treatment was able to recover this OPN response by $52 \% \pm 1 \%$, compared to IFT88 siRNA and vehicle treated cells. This further suggests that the length of primary cilia is critical to their function as a mechanosensor and that as cilia formation was restored, so was mechanosensitivity too. Then we confirmed that fenoldopam treatment of healthy, ciliated cells had no significant effect on cilia incidence (Fig. 4D) or IFT88 mRNA expression (Fig. 4E).

Finally, we examined a potential molecular pathway through which fenoldopam increases primary cilia length. cAMP has been previously shown to be involved in ciliogenesis and primary cilia-mediated mechanotransduction, so we quantified adenylyl cyclase activity by measuring stimulated cAMP production (Besschetnova et al., 2010; Kwon et al., 2010). We increased primary cilia length by fenoldopam treatment and then briefly stimulated the cells with the adenylyl cyclase agonist forskolin (Fig. 5A). Fenoldopam treatment significantly enhanced the forskolin stimulated cAMP response by $130 \% \pm 25 \%$ compared to vehicle control. Additionally, fenoldopam treatment induced a $20 \% \pm 8 \%$ increase in AC6 mRNA expression (Fig. 5B). Because of the significant role of AC6 in primary cilia-mediated mechanotransduction, we used inhibition of AC6 as an alternative model of impaired cell mechanosensitivity. Treatment of osteocytes with AC6 siRNA resulted in a small but significant decrease in cilia length by $10 \% \pm 3 \%$ (Fig. 5C), while AC6 inhibition had no effect on cilia incidence (Fig. 5D). Fenoldopam had no effect on recovering cilia length or incidence in AC6 siRNA treated cells. When cells with diminished AC6 were mechanically stimulated, AC6 knockdown cells displayed decreased AC6 and flow-induced OPN expression by $50 \% \pm 3 \%$ and $30 \% \pm 3 \%$, respectively, which was not recovered with fenoldopam treatment (Fig. 5E, F).

\section{Discussion}

Our results demonstrated that primary cilia length plays a significant role in cell mechanosensitivity. We employed two distinct, clinically utilised, small molecules to increase cilia length and both resulted in enhanced mechanosensitivity. Cells with impaired ciliogenesis had impaired mechanosensing, but this could be recovered with fenoldopam treatment. Finally, we showed that fenoldopam modulates osteocyte mechanosensitivity through a mechanism involving AC6 and cells with diminished AC6 had shorter cilia and impaired mechanosensing.

Based on clinical and biochemical considerations, fenoldopam was a more suitable candidate for further study than lithium and thus was only used for studying the effects of IFT88 and AC6 knockdowns. Fenoldopam is a dopamine D1-like receptor agonist clinically used as a vasodilator in cases of extreme hypertension (Murphy et al., 2001; Post and Frishman, 1998). Lithium has a much less defined function and is clinically used to treat a wide range of mental disorders, including bipolar disorder (Marmol, 2008). Furthermore, lithium is an inhibitor of GSK-3 $\beta$ and can have downstream effects on various signalling pathways including Wnt and Hedgehog. While lithium has been used to increase cilia length in a variety of cell types, other GSK-3 $\beta$ inhibitors have no effect on cilia length (Jope, 2003; Ou et al., 2009).

Fenoldopam treatment increased cilia length, but also plays a role in adenylyl cyclase activity. The increase in forskolin stimulated adenylyl cyclase activity with fenoldopam treatment implicates two potential mechanisms. First, it is possible that fenoldopam sensitises adenylyl cyclases, resulting in an increased cAMP response to forskolin. Alternatively, fenoldopam may increase production of adenylyl cyclases, augmenting forskolin stimulated cAMP production. This second notion is consistent with previous work indicating that fenoldopam treatment upregulates AC6, a specific adenylyl cyclase isoform, production in kidney cells (Yu et al., 2014). Our results support this possible molecular pathway, demonstrating an increase in AC6 mRNA expression in response to fenoldopam stimulation. Previously, we have demonstrated that AC6 localises to the osteocyte primary cilium and is critical for primary cilia-mediated mechanotransduction (Kwon et al., 2010). Besschetnova et al. reported that stimulating the cAMP signalling pathway results in increased cilia length (Besschetnova et al., 2010). Using an siRNA-mediated knockdown, they showed that AC6 has a functional role in mediating primary cilia elongation. Together, these findings indicate that AC6-cAMP dynamics are critical to both primary cilia length and mechanotransduction and that fenoldopam treatment stimulates this pathway. Furthermore, these results suggest that fenoldopam treatment enhances ciliary protein production to promote cilium elongation.

Adenylyl cyclases and cAMP contribute to recover and elongate primary cilia by stimulating IFT particle transport. It has previously been reported that stimulation of the adenylyl cyclase-cAMP-PKA signalling pathway augments anterograde transport of IFT particles to promote cilia elongation (Besschetnova et al., 2010). Because fenoldopam enhances adenylyl cyclase production, this suggests that fenoldopam treatment is potentiating adenylyl cyclase activity and IFT particle transport. Our model of impaired cilia utilised an IFT88 knockdown, not a complete knockout of the gene, so it is possible that fenoldopam was able to enhance remaining IFT88 function and promote cilia elongation and rescue cilia incidence. Furthermore, this presupposes that even though the IFT88 knockdown is satisfactory to impair cilia formation and function, sufficient IFT88 remains to elongate cilia. Our data show no change in IFT88 mRNA expression elicited by fenoldopam treatment suggesting that fenoldopam stimulated the remaining IFT88, rather than promoting 
production of new IFT88. However, this does not discount the notion that fenoldopam treatment may instead prevent IFT88 knockdown driven disassembly of the cilium.

The specific means by which cells with longer cilia are more mechanosensitive remains elusive, but there are two potential mechanisms of how this may occur. Schwartz et al. developed one of the first models of primary cilia deflection under fluid flow and hypothesised that longer cilia would experience greater membrane strain to increase opening of stretch-activated ion channels on the ciliary membrane (Schwartz et al., 1997). Alternatively, longer cilia may simply allow for the presence of more ciliaspecific proteins and signalling molecules within this microdomain (Breslow et al., 2013; Kee et al., 2012). Increasing the total amount of ciliary protein could enhance signal transduction within the ciliary compartment, modifying primary cilia-mediated mechanosensitivity. In fact, fenoldopam treated cells exposed to fluid flow have increased ciliary influx of calcium, which has been identified as one initiator of the mechanotransduction signalling cascade (Jin et al., 2014; Yuan et al., 2015). It is also possible that cilium-lengthening agents actually enhance ciliary protein production and trafficking to promote cilium elongation.

The correlation between cilia length and critical ciliary proteins involved in mechanosensing was examined with AC6 siRNA treatment. The knockdown of AC6 decreased flow-induced osteogenic gene expression, yet fenoldopam treatment was not sufficient to rescue AC6 expression or OPN expression as was demonstrated in the IFT88 knockdown model. Because fenoldopam treatment was not able to recover AC6 or OPN mRNA expression in AC6 knockdown cells, this may suggest that the ability of fenoldopam to enhance AC6 activity is critical for recovering cellular mechanosensing. However, AC6 knockdown also decreased primary cilia length, which was not recovered with fenoldopam and did not alter cilia incidence. Altogether, these data suggest that both cilia length and protein production may be critical in primary cilia-mediated mechanosensing.

Cells with longer cilia are more mechanosensitive, but primary cilia cannot be elongated indefinitely. Longer cilia are exposed to greater drag force and are more likely to be sheared off (Hierck et al., 2008). For example, endothelial cell primary cilia are flow sensors in regions of low shear, specifically because they are cleaved off as shear stresses increase (Van der Heiden et al., 2008). Interestingly, electron microscopy has shown that primary cilia structure is not constant along the ciliary axoneme and becomes increasingly disorganised and asymmetric at the distal tip (Odor and Blandau, 1985; Yamamoto and Kataoka, 1986). This loss of microtubule symmetry reduces the bending stiffness of the cilium at the distal end, making drastically elongated cilia more susceptible to removal by fluid shear (Rydholm et al., 2010).

While osteocyte primary cilia are free-standing flow sensors in vitro, their mechanosensing function may differ in vivo. It has been estimated that the lacunar space in which osteocytes reside in vivo allows for only a $1 \mu \mathrm{m}$ long cilium (McNamara et al., 2009; Uzbekov et al., 2012). Due to the spatial limitations within the lacuna, the potential effect of pharmacologically enhancing osteocyte cilia length in vivo is unclear. In fact, these spatial constraints may point to the cilium not being a free-flowing mechanosensor at all. Rather, osteocyte cilia may anchor to the lacunar wall, similarly to chondrocyte primary cilia, which form integrin attachments with the surrounding extracellular matrix, ECM (McGlashan et al., 2006). A computational model by Vaughan et al. simulated osteocytes exposed to fluid flow within the lacunar-canalicular network (Vaughan et al., 2014). The authors modelled a free-standing cilium, $0.5 \mu \mathrm{m}$ long, within a lacuna and calculated the resulting strain at the base of the cilium. Their model suggests that a cilium in this configuration does not experience a great enough strain to function as a flow sensor, but a cilium directly attached to the ECM does. However, the authors do not account for the amount of membrane strain necessary to stimulate stretch-activated ion channels on the ciliary membrane and may have overestimated the required strain for cilium stimulation. Fenoldopam treatment not only increases length, but may also enhance mechanosensitive protein levels, such as adenylyl cyclases and ion channels, within the cilium. Regardless of cilium length, this enriched protein trafficking to the cilium would increase chemical kinetics within the ciliary microdomain to modify cellular mechanosensitivity.

Our results certainly suggest that fenoldopam treatment or similar agents, which may increase cilia length, can be a potent method to enhance cell mechanosensitivity. However, the effect of fenoldopam on whole bone has not been examined, so the potential skeletal and systemic consequences in translating this work to in vivo models is still unknown. Statistical analysis of patients treated with various antidepressants, such as selective serotonin reuptake inhibitors, which can block dopamine D2 receptors, revealed that these patients have increased risk of hip and femur fracture (Van Den Brand et al., 2009; Smith et al., 2009). Furthermore, many antidepressants and antipsychotics that block dopamine D2 receptors can cause decreased oestrogen and testosterone levels leading to significantly reduced bone mass (Phillip and Lazar, 2003). All of these studies examine treatments which may affect dopamine D2 receptors, but at present there are no examinations of the impact of dopamine D1 receptor agonists on bone formation. As such, no connection between fenoldopam treatment and skeletal health has been established. Because of this lack of direct evidence, such drugs have the potential for a wide range of unknown side effects. Further work needs to be performed before any drugs altering dopamine signalling are used to treat bone disease.

Targeting primary cilia-mediated mechanotransduction has widespread applications in preventative medicine that reach far beyond osteocytes. Numerous diseases are characterised by impaired primary cilia function. Mutations of PC2, polycystin 2, are attributed to polycystic kidney disease and skeletal deformations. Bardet-Biedl syndrome is characterised by malfunctioning BBS proteins at the base of the primary cilium, causing retinopathy, polydactyly and renal failure (Loktev et al., 2008; Mochizuki et al., 1996; Xiao et al., 2011). Recently, primary cilia have even been implicated in tumour development. Primary 
cilia help regulate Wnt signalling, changes in which have been correlated with cancer cell progression (Lancaster et al., 2011). Furthermore, some cancer cell types lose their primary cilia, which potentially contributes to their insensitivity to repressive signals (Plotnikova et al., 2008). Additionally, atherosclerotic plaques form in areas of reduced and disturbed arterial fluid flow, regions that interestingly have an increased incidence of primary cilia. This suggests that these cells are compensating, increasing their sensitivity to reduced fluid flow in order to promote an adequate cellular response (Van der Heiden et al., 2008; Warboys et al., 2011). Within bone, osteocytes utilise primary cilia to sense and respond to mechanical cues. In vitro and in vivo studies demonstrate that when these cilia are removed there is a decreased bone formation response to loading (Kwon et al., 2010; Malone et al., 2007; Temiyasathit et al., 2012). Fenoldopam is already an FDA approved drug and our results point to it being an attractive candidate for study in numerous in vitro and in vivo applications to treat such a myriad of conditions.

\section{Conclusion}

We have demonstrated that cells with longer primary cilia are more mechanosensitive and we present a simple, yet robust, method to enhance primary cilia-mediated mechanotransduction. Fenoldopam treatment not only increased primary cilia length, protein production and mechanosensitivity, but also rescued cilia form and function in cells with impaired cilia. Although fenoldopam and lithium are likely not ideal treatments for all ciliarelated conditions, the strategy of modulating primary cilia sensitivity may aid in combating the phenotypes displayed by various ciliopathies, maintain sensitivity of cancer cells so that they respond to repressive signals, cue cellular responses to slow atherosclerotic plaque formation and stimulate load-induced bone formation.

\section{Acknowledgements}

We would like to acknowledge Emily Moore and Michael Duffy for their valuable discussions and input in regards to this work. This work was supported by National Institute of Arthritis and Musculoskeletal and Skin Diseases/National Institute of Health (NIAMS/NIH) grant AR062177.

We wish to confirm that there are no known conflicts of interest associated with this publication and there has been no significant financial support for this work that could have influenced its outcome.

\section{References}

Arnsdorf EJ, Tummala P, Kwon RY, Jacobs CR (2009) Mechanically induced osteogenic differentiation-the role of RhoA, ROCKII and cytoskeletal dynamics. J Cell Sci 122: 546-553.
Besschetnova TY, Kolpakova-Hart E, Guan Y, Zhou J, Olsen BR, Shah JV (2010) Identification of signaling pathways regulating primary cilium length and flowmediated adaptation. Curr Biol 20: 182-187.

Bivi N, Pacheco-Costa R, Brun LR, Murphy TR, Farlow NR, Robling AG, Bellido T, Plotkin LI (2013) Absence of Cx43 selectively from osteocytes enhances responsiveness to mechanical force in mice. J Orthop Res 31: 1075-1081.

Bliziotes M, McLoughlin S, Gunness M, Fumagalli F, Jones SR, Caron MG (2000) Bone histomorphometric and biomechanical abnormalities in mice homozygous for deletion of the dopamine transporter gene. Bone 26: 15-19.

Breslow DK, Koslover EF, Seydel F, Spakowitz AJ, Nachury MV (2013) An in vitro assay for entry into cilia reveals unique properties of the soluble diffusion barrier. J Cell Biol 203: 129-147.

Burra S, Nicolella DP, Francis WL, Freitas CJ, Mueschke NJ, Poole K, Jiang JX (2010) Dendritic processes of osteocytes are mechanotransducers that induce the opening of hemichannels. Proc Natl Acad Sci U S A 107: 13648-13653.

Castillo AB, Blundo JT, Chen JC, Lee KL, Yereddi NR, Jang E, Kumar S, Tang WJ, Zarrin S, Kim JB, Jacobs CR (2012) Focal adhesion kinase plays a role in osteoblast mechanotransduction in vitro but does not affect loadinduced bone formation in vivo. PLoS One 7: 1-11.

Clément-Lacroix P, Ai M, Morvan F, Roman-Roman S, Vayssière B, Belleville C, Estrera K, Warman ML, Baron R, Rawadi G (2005) Lrp5-independent activation of Wnt signaling by lithium chloride increases bone formation and bone mass in mice. Proc Natl Acad Sci U S A 102: 17406-17411.

Drago J, Gerfen CR, Lachowicz JE, Steiner H, Hollon TR, Love PE, Ooi GT, Grinberg A, Lee EJ, Huang SP (1994) Altered striatal function in a mutant mouse lacking D1A dopamine receptors. Proc Natl Acad Sci U S A 91: 12564-12568.

Ehrlich P, Lanyon L (2002) Mechanical strain and bone cell function: a review. Osteoporos Int 9: 688-700.

Fujihara S, Yokozeki M, Oba Y, Higashibata Y, Nomura S, Moriyama K (2006) Function and regulation of osteopontin in response to mechanical stress. J Bone Min Res 21: 956-964.

Geiger B, Bershadsky A, Pankov R, Yamada KM (2001) Transmembrane crosstalk between the extracellular matrix--cytoskeleton crosstalk. Nat Rev Mol Cell Biol 2: 793-805.

Hierck BP, Van der Heiden K, Alkemade FE, Van de Pas S, Van Thienen J V, Groenendijk BC, Bax WH, Van der Laarse A, Deruiter MC, Horrevoets AJ, Poelmann RE (2008) Primary cilia sensitize endothelial cells for fluid shear stress. Dev Dyn 237: 725-735.

Jin X, Mohieldin AM, Muntean BS, Green JA, Shah J V, Mykytyn K, Nauli SM (2014) Cilioplasm is a cellular compartment for calcium signaling in response to mechanical and chemical stimuli. Cell Mol Life Sci 71: 2165-2178.

Jope RS (2003) Lithium and GSK-3: One inhibitor, two inhibitory actions, multiple outcomes. Trends Pharmacol Sci 24: 441-443. 
Kathem SH, Mohieldin AM, Abdul-Majeed S, Ismail SH, Altaei QH, Alshimmari IK, Alsaidi MM, Khammas H, Nauli AM, Joe B, Nauli SM (2014) Ciliotherapy: a novel intervention in polycystic kidney disease. J Geriatr Cardiol 11: 63-73.

Kee HL, Dishinger JF, Lynne Blasius T, Liu C-J, Margolis B, Verhey KJ (2012) A size-exclusion permeability barrier and nucleoporins characterize a ciliary pore complex that regulates transport into cilia. Nat. Cell Biol. 14: 431-437.

Klein-Nulend J, Burger EH, Semeins CM, Raisz LG, Pilbeam CC (1997) Pulsating fluid flow stimulates prostaglandin release and inducible prostaglandin $\mathrm{G} / \mathrm{H}$ synthase mRNA expression in primary mouse bone cells. J Bone Miner Res 12: 45-51.

$\mathrm{Ku}$ DN (1997) Blood flow in arteries. Annu Rev Fluid Mech 29: 399-434.

Kwon RY, Temiyasathit S, Tummala P, Quah CC, Jacobs CR (2010) Primary cilium-dependent mechanosensing is mediated by adenylyl cyclase 6 and cyclic AMP in bone cells. FASEB J 24: 2859-68.

Lancaster MA, Schroth J, Gleeson JG (2011) Subcellular spatial regulation of canonical Wnt signalling at the primary cilium. Nat Cell Biol 13: 700-707.

Lee DJ, Tseng HC, Wong SW, Wang Z, Deng M, Ko C-C (2015a) Dopaminergic effects on in vitro osteogenesis. Bone Res 3: 15020.

Lee KL, Hoey DA, Spasic M, Tang T, Hammond HK, Jacobs CR (2014) Adenylyl cyclase 6 mediates loadinginduced bone adaptation in vivo. FASEB J. 28: 1157-1165.

Lee KL, Guevarra MD, Nguyen AM, Chua MC, Wang Y, Jacobs CR (2015b) The primary cilium functions as a mechanical and calcium signaling nexus. Cilia 4: 7.

Lehman JM, Michaud EJ, Schoeb TR, Aydin-Son Y, Miller M, Yoder BK (2008) The oak ridge polycystic kidney mouse: modeling ciliopathies of mice and men. Dev Dyn 237: 1960-1971.

Liu W, Xu S, Woda C, Kim P, Weinbaum S, Satlin LM (2003) Effect of flow and stretch on the $[\mathrm{Ca} 2+]$ i response of principal and intercalated cells in cortical collecting duct. Am J Physiol Renal Physiol 285: F998-F1012.

Loktev A V, Zhang Q, Beck JS, Searby CC, Scheetz TE, Bazan JF, Slusarski DC, Sheffield VC, Jackson PK, Nachury M V (2008) A BBSome subunit links ciliogenesis, microtubule stability, and acetylation. Dev Cell 15: 854865 .

Malone AMD, Anderson CT, Tummala P, Kwon RY, Johnston TR, Stearns T, Jacobs CR (2007) Primary cilia mediate mechanosensing in bone cells by a calciumindependent mechanism. Proc Natl Acad Sci U S A 104: 13325-13330.

Mantila Roosa SM, Liu Y, Turner CH (2011) Gene expression patterns in bone following mechanical loading. J Bone Miner Res 26: 100-112.

Marmol F (2008) Lithium: bipolar disorder and neurodegenerative diseases possible cellular mechanisms of the therapeutic effects of lithium. Prog NeuroPsychopharmacol Biol Psychiatry 32: 1761-1771.

McGlashan SR, Jensen CG, Poole CA (2006) Localisation of extracellular matrix receptors on the chondrocyte primary cilium. J Histochem Cytochem 54: 1005-1014.
McNamara LM, Majeska RJ, Weinbaum S, Friedrich V, Schaffler MB (2009) Attachment of osteocyte cell processes to the bone matrix. Anat Rec 292: 355-363.

Miyoshi K, Kasahara K, Miyazaki I, Asanuma M (2009) Lithium treatment elongates primary cilia in the mouse brain and in cultured cells. Biochem Biophys Res Commun 388: 757-762.

Mochizuki T, Wu G, Hayashi T, Xenophontos SL, Veldhuisen B, Saris JJ, Reynolds DM, Cai Y, Gabow PA, Pierides A, Kimberling WJ, Breuning MH, Deltas CC, Peters DJ, Somlo S (1996) PKD2, a gene for polycystic kidney disease that encodes an integral membrane protein. Science 272: 1339-1342.

Murphy MB, Murray C, Shorten GD (2001) Fenoldopam: a selective peripheral dopamine-receptor agonist for the treatment of severe hypertension. N Engl J Med 345: 1548-1557.

Norvell SM, Ponik SM, Bowen DK, Gerard R, Pavalko FM (2004) Fluid shear stress induction of COX-2 protein and prostaglandin release in cultured MC3T3-E1 osteoblasts does not require intact microfilaments or microtubules. J Appl Physiol 96: 957-966.

Odor DL, Blandau RJ (1985) Observations on the solitary cilium of rabbit oviductal epithelium: its motility and ultrastructure. Am J Anat 174: 437-453.

Ou Y, Ruan Y, Cheng M, Moser JJ, Rattner JB, van der Hoorn FA. (2009) Adenylate cyclase regulates elongation of mammalian primary cilia. Exp.Cell Res 315: 2802-2817.

Pazour GJ, Dickert BL, Vucica Y, Seeley ES, Rosenbaum JL, Witman GB, Cole DG (2000) Chlamydomonas IFT88 and its mouse homologue, polycystic kidney disease gene $\mathrm{Tg} 737$, are required for assembly of cilia and flagella. J. Cell Biol. 151: 709-718.

Phan MN, Leddy HA, Votta BJ, Kumar S, Levy DS, Lipshutz DB, Suk HL, Liedtke W, Guilak F (2009) Functional characterisation of TRPV4 as an osmotically sensitive ion channel in porcine articular chondrocytes. Arthritis Rheum 60: 3028-3037.

Phillip M, Lazar L (2003) The regulatory effect of hormones and growth factors on the pubertal growth spurt. Endocrinologist 13: 465-469.

Piperi C (2015) Polycystins and mechanotransduction: from physiology to disease. World J Exp Med 5: 200.

Plotkin LI, Speacht TL, Donahue HJ (2015) Cx43 and mechanotransduction in bone. Curr Osteoporos Rep 13: 67-72.

Plotnikova OV, Golemis EA, Pugacheva EN (2008) Cell cycle-dependent ciliogenesis and cancer. Cancer Res 68: 2058-2061.

Post JB, Frishman WH (1998) Fenoldopam: a new dopamine agonist for the treatment of hypertensive urgencies and emergencies. J Clin Pharmacol 38: 2-13.

Praetorius HA, Spring KR (2001) Bending the MDCK cell primary cilium increases intracellular calcium. J Membr Biol 184: 71-79.

Praetorius HA, Spring KR (2003) Removal of the MDCK cell primary cilium abolishes flow sensing. J Membr Biol 191: 69-76.

Raisz LG (1999) Prostaglandins and bone: physiology and pathophysiology. Osteoarthr Cartil 7: 419-421. 
Rydholm S, Zwartz G, Kowalewski JM, Kamali-Zare P, Frisk T, Brismar H (2010) Mechanical properties of primary cilia regulate the response to fluid flow. Am J Physiol Renal Physiol 298: F1096-102.

Schwartz EA, Leonard ML, Bizios R, Bowser SS (1997) Analysis and modeling of the primary cilium bending response to fluid shear. Am J Physiol 272: F132-F138.

Smith GS, Ma Y, Dhawan V, Chaly T, Eidelberg D (2009) Selective serotonin reuptake inhibitor (SSRI) modulation of striatal dopamine measured with [ $11 \mathrm{C}$ raclopride and positron emission tomography. Synapse 63: 1-6.

Temiyasathit S, Tang WJ, Leucht P, Anderson CT, Monica SD, Castillo AB, Helms JA, Stearns T, Jacobs CR (2012) Mechanosensing by the primary cilium: deletion of Kif3a reduces bone formation due to loading. PLoS One 7: e33368.

Tonna EA, Lampen NM (1972) Electron microscopy of aging skeletal cells. I. Centrioles and solitary cilia. J Gerontol 27: 316-324.

Upadhyay VS, Muntean BS, Kathem SH, Hwang JJ, Aboualaiwi WA, Nauli SM (2014) Roles of dopamine receptor on chemosensory and mechanosensory primary cilia in renal epithelial cells. Front Physiol 5: 72.

Uzbekov RE, Maurel DB, Aveline PC, Pallu S, Benhamou CL, Rochefort GY (2012) Centrosome fine ultrastructure of the osteocyte mechanosensitive primary cilium. Microsc Microanal 18: 1430-1441.

Van Den Brand MWM, Samson MM, Pouwels S, Van Staa TP, Thio B, Cooper C, Leufkens HGM, Egberts ACG, Verhaar HJJ, De Vries F (2009) Use of anti-depressants and the risk of fracture of the hip or femur. Osteoporos Int 20: 1705-1713.

Van der Heiden K, Hierck BP, Krams R, de Crom R, Cheng C, Baiker M, Pourquie MJBM, Alkemade FE, DeRuiter MC, Gittenberger-de Groot AC, Poelmann RE (2008) Endothelial primary cilia in areas of disturbed flow are at the base of atherosclerosis. Atherosclerosis 196: 542-550.

Vaughan TJ, Mullen CA, Verbruggen SW, McNamara LM (2014) Bone cell mechanosensation of fluid flow stimulation: a fluid-structure interaction model characterising the role integrin attachments and primary cilia. Biomech Model Mechanobiol 14: 703-718.

Warboys CM, Amini N, de Luca A, Evans PC (2011) The role of blood flow in determining the sites of atherosclerotic plaques. F1000 Med Rep 3: 5.

Xiao Z, Dallas M, Qiu N, Nicolella D, Cao L, Johnson M, Bonewald L, Quarles LD (2011) Conditional deletion of Pkd1 in osteocytes disrupts skeletal mechanosensing in mice. FASEB J 25: 2418-2432.

Yamamoto M, Kataoka K (1986) Electron microscopic observation of the primary cilium in the pancreatic islets. Arch Histol Jpn 49: 449-457.
Yoder BK, Tousson A, Millican L, Wu JH, Bugg CE, Schafer JA, Balkovetz DF (2002) Polaris, a protein disrupted in orpk mutant mice, is required for assembly of renal cilium. Am J Physiol Renal Physiol 282: F541-F552.

You L, Temiyasathit S, Lee P, Kim CH, Tummala P, Yao W, Kingery W, Malone AM, Kwon RY, Jacobs CR (2008) Osteocytes as mechanosensors in the inhibition of bone resorption due to mechanical loading. Bone 42: 172-179.

Yu P, Sun M, Villar VAM, Zhang Y, Weinman EJ, Felder RA, Jose PA (2014) Differential dopamine receptor subtype regulation of adenylyl cyclases in lipid rafts in human embryonic kidney and renal proximal tubule cells. Cell Signal 26: 2521-2529.

Yuan S, Zhao L, Brueckner M, Sun Z (2015) Intraciliary calcium oscillations initiate vertebrate left-right asymmetry. Curr Biol 25: 556-567.

\section{Discussion with Reviewers}

Rosa Serra: How does the author envision a clinical use for bone density disorders? Would the drug be used in combination with exercise?

Authors: While this work is entirely in vitro and at this point it is difficult to definitively make any conclusions about in vivo translation, these findings are suggestive of a pro-osteogenic therapeutic strategy. Our work posits that fenoldopam sensitises bone cells to mechanical stimulation, so that when a patient receives a similar treatment, exercise-induced bone adaptation would be augmented. Thus, any potential treatment regimen would likely include some exercise component in treating bone density disorders.

Fredrick Pavalko: How do localised changes in cAMP at the primary cilia lead to global changes in bone density? Authors: Adenylyl cyclases convert ATP to cAMP, which activates PKA to regulate CREB-mediated transcriptional modification. Previous work from our group demonstrated that AC6, a specific isoform of adenylyl cyclase known to localise to cilia, plays a critical role in load-induced bone adaptation. Specifically, mice lacking AC6 display a significantly abrogated response to compressive ulnar load (Lee KL et al., 2014). It is possible that primary ciliamediated mechanotransduction is initiated in the cilium, causing a change in ciliary and cytosolic cAMP, which modifies downstream gene transcription. In osteocytes, this change in gene expression likely alters intercellular signalling to osteoblasts, osteoclasts and progenitor cells to dictate bone formation, density and overall skeletal health.

Editor's note: The Scientific Editor responsible for this paper was Juerg Gasser. 